\title{
RENDIMENTO DE MASSA SECA DE Physalis angulata L. SOB PROPORÇÕES DE NITRATO E AMÔNIO EM CULTIVO HIDROPÔNICO
}

Alismário Leite da Silva ${ }^{1}$, Marilza Neves do Nascimento ${ }^{2}$, Tamara Torres Tanan ${ }^{3}$, Romeu da Silva Leite ${ }^{4}$ e Uasley Caldas de Oliveira ${ }^{5}$

${ }^{1}$ Graduando em Agronomia, Bolsista PROBIC/UEFS, Universidade Estadual de Feira de Santana (UEFS), Feira de Santana, Brasil. (alismarioagronomo@hotmail.com)

${ }^{2}$ Professora Doutora do Curso de Agronomia, Universidade Estadual de Feira de Santana (UEFS), Feira de Santana, Brasil

${ }^{3}$ Doutoranda em Recursos Genéticos Vegetais pela Universidade Estadual de Feira de Santana (UEFS), Feira de Santana, Brasil

${ }^{4}$ Mestrando em Recursos Genéticos Vegetais, Universidade Estadual de Feira de Santana (UEFS), Feira de Santana, Brasil

${ }^{5}$ Mestrando em Solos e Qualidade de Ecossistemas, Universidade Federal do Recôncavo Baiano (UFRB), Cruz das Almas, Brasil

Recebido em: 06/04/2018 - Aprovado em: 10/06/2018 - Publicado em: 20/06/2018 DOI: 10.18677/EnciBio_2018A74

\begin{abstract}
RESUMO
As principais fontes de absorção de $\mathrm{N}$ pelas plantas são os íons $\mathrm{NH}_{4}^{+}$e $\mathrm{NO}_{3}{ }^{-} \mathrm{e}$ a utilização de proporções desbalanceadas pode promover alteração no crescimento e desenvolvimento de diversas espécies. Dessa forma, objetivou-se com esse trabalho avaliar o efeito de proporções de $\mathrm{NO}_{3}{ }^{-}: \mathrm{NH}_{4}{ }^{+}$em solução nutritiva na produção de massa seca de plantas de Physalis angulata L. O trabalho foi conduzido em casa de vegetação na Unidade Experimental Horto Florestal da Universidade Estadual de Feira de Santana (UEFS), Feira de Santana, Bahia, em sistema de cultivo hidropônico. Os tratamentos foram distribuídos nas seguintes proporções $\left(\mathrm{NO}_{3}{ }^{-}: \mathrm{NH}_{4}{ }^{+}\right): 100: 0 ; 75: 25 ; 50: 50 ; 25: 75$. Foram avaliadas as seguintes variáveis: massa seca da raiz, do caule, das folhas e dos frutos e a área foliar. Além disso, foi calculada a massa seca da parte aérea e a relação raiz parte aérea. Foi verificado que a aplicação de $75 \%$ de amônio na solução provocou redução na massa seca das plantas de $P$. angulata. As proporções com 0,25 e $50 \%$ de amônio não interferiram na produção de massa seca de todas as partes das plantas de $P$. angulata, podendo ser utilizada até $50 \%$ de amônio na solução nutritiva para o cultivo da espécie.
\end{abstract}

PALAVRAS-CHAVE: Adubação Nitrogenada, hidroponia, camapú.

\section{DRY MASS YIELD OF Physalis angulata L. UNDER PROPORTIONS OF NITRATE AND AMMONIA IN HYDROPONIC CROP}

\footnotetext{
ABSTRACT

The main sources of $\mathrm{N}$ uptake by plants are the $\mathrm{NH}_{4}{ }^{+}$and $\mathrm{NO}_{3}{ }^{-}$ions and the use of unbalanced proportions can promote alteration in the growth and development of several species. The objective of this work was to evaluate the effect of $\mathrm{NO}_{3}{ }^{-}: \mathrm{NH}_{4}{ }^{+}$ ratios in nutrient solution on the production of dry mass of Physalis angulata $\mathrm{L}$. ENCICLOPÉDIA BIOSFERA, Centro Científico Conhecer - Goiânia, v.15 n.27; p. 31 2018
} 
plants. The work was conducted in a greenhouse at the Horto Florestal Experimental Unit of the State University of Feira de Santana (UEFS), Feira de Santana, Bahia, under a hydroponic cultivation system. Treatments were distributed in the following proportions $\left(\mathrm{NO}_{3}{ }^{-} \mathrm{NH}_{4}{ }^{+}\right)$100:0; 75:25; 50:50; 25:75. The variables were evaluated: root dry mass, stem dry mass, leaves dry mass and fruits dry mass, and leaf area (AF). In addition, the dry shoot mass and the root shoot ratio were calculated. The application of $75 \%$ ammonium in the solution causes reduction in the dry mass of the plants of $P$. angulata. The proportions with $0,25 \%$ and $50 \%$ of ammonium didn't interfere in the production of dry mass of all parts of the plants, up to $50 \%$ of ammonium can be used in the nutrient solution for the cultivation of the species.

KEYWORDS: Nitrogen fertilization, Hydroponic, camapú.

\section{INTRODUÇÃO}

O gênero Physalis possui em torno de 90 espécies distribuídas no continente americano, das quais 8 podem ser encontradas no Brasil, mais precisamente na Amazônia e no Nordeste (STEHMANN et al., 2015). Mesmo com a crescente popularidade de espécies como a Physalis angulata L. ( $P$. angulata), ainda são escassas as informações sobre a produção, consumo e comercialização no Brasil (LIMA et al., 2013).

A $P$. angulata é também conhecida como, "camapú", "mullaca" ou "juá-decapote", sendo encontrada em algumas regiões do Brasil, mais precisamente nas regiões Norte e Nordeste (LIMA et al., 2013). Sua produção é uma alternativa de grande viabilidade para pequenos produtores rurais, bem como para a agricultura familiar, pois apresenta alto potencial para uso medicinal (CRUZ et al., 2015), além disso, pode trazer um retorno econômico devido a possibilidade de consumo dos seus frutos exóticos, o que poderá agregar ainda mais o valor na sua comercialização. A maior parte dos frutos encontrados nos supermercados brasileiros advém da Colômbia, visto que a produção comercial no Brasil ainda é pouco difundida (CRUZ et al., 2015).

Estudos sobre o cultivo da espécie vêm sendo realizados no nordeste, alcançando resultados satisfatórios para seu cultivo nas condições dessa região. Leite et al. (2017) avaliaram a eficiência no cultivo de $P$. angulata em sistema hidropônico, e definiu a dose ideal de nitrogênio $(\mathrm{N})$ para melhor produção econômica da espécie. A adubação nitrogenada é uma das práticas agronômicas que mais interferem na produtividade das plantas (STEFEN et al., 2015), mas a correta escolha da sua fonte é um dos principais temas de estudos, pois as plantas podem responder de várias maneiras as diferentes formas de $\mathrm{N}$, sendo absorvidas pelas raízes preferencialmente nas formas de nitrato $\left(\mathrm{NO}_{3}{ }^{-}\right)$ou amônio $\left(\mathrm{NH}_{4}{ }^{+}\right)$ (MARTINEZ-ANDÚJAR et al., 2013).

De acordo com Esteban et al. (2016), o amônio pode limitar o crescimento das plantas, pois a sua utilização, como única fonte de nitrogênio, pode ocasionar problemas morfológicos e fisiológicos, reduzindo assim o seu crescimento e causando toxidez às plantas. Por outro lado, há um menor consumo de energia, devido a sua inserção direta na cadeia de carbono na assimilação de nitrogênio, não sendo necessária as fases de redução pela ação enzimática, com gasto de energia, como ocorre para o nitrato (BITTSÁNSZKY et al., 2015), podendo assim, aumentar a eficiência do uso desse nutriente (SARASKETA et al., 2014).

A maioria das culturas absorve preferencialmente o $\mathrm{NO}_{3}{ }^{-}$do que $\mathrm{NH}_{4}{ }^{+} \mathrm{e}$, em sistemas hidropônicos, a forma nítrica é a mais utilizada para o fornecimento de 
nitrogênio, o que pode ocasionar o acúmulo de $\mathrm{NO}_{3}^{-}$e nitrito $\left(\mathrm{NO}_{2}{ }^{-}\right)$em níveis elevados, podendo causar problemas aos seres humanos (CHANG et al., 2013). A resposta da aplicação das formas de $\mathrm{N}$ nas plantas é muito variada, havendo diferenças quanto ao crescimento e desenvolvimento em diferentes espécies (HUANG et al., 2013). Essas formas podem ser diferenciadas com relação aos custos de sua aplicação, pelo potencial de volatilização e lixiviação, pelo processo de acidificação no solo e principalmente, pela absorção das plantas (MAROUELLI et al., 2014).

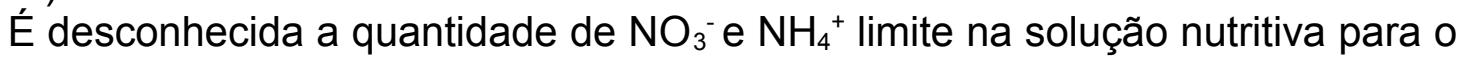
pleno desenvolvimento da cultura da $P$. angulata. Dessa forma, o presente trabalho teve como objetivo avaliar o efeito de proporções de $\mathrm{NO}_{3}{ }^{-}: \mathrm{NH}_{4}{ }^{+}$em solução nutritiva no crescimento de $P$. angulata.

\section{MATERIAL E MÉTODOS}

O trabalho foi conduzido na Unidade Experimental Horto Florestal da Universidade Estadual de Feira de Santana (UEFS), Feira de Santana, Bahia. As sementes de $P$. angulata foram colocadas para germinar em bandejas com substrato comercial sob irrigação diária, mantendo-as sempre úmida. Vinte dias após a germinação, as mudas foram selecionadas quanto à uniformidade da parte aérea e radicular e transplantadas para o sistema hidropônico tipo floating, em vasos individuais. Foi utilizada a solução de Sarruge (1975) modificada para o cultivo de $P$. angulata com o uso da dose de $162 \mathrm{mg} \cdot \mathrm{L}^{-1}$ de nitrogênio (LEITE et al., 2017). O pH da solução nutritiva foi medido diariamente e ajustado em 6,5 , quando necessário. A condutividade elétrica (CE) foi mantida entre 60 a $100 \%$ da inicial, com a reposição de água sempre que necessário e reposição da solução.

O delineamento utilizado foi 0 inteiramente casualizado, com quatro tratamentos e nove repetições. Os tratamentos foram constituídos de quatro proporções de $\mathrm{NO}_{3}{ }^{-}: \mathrm{NH}_{4}{ }^{+}(100: 0 ; 75: 25 ; 50: 50,25: 75)$. As avaliações foram realizadas 45 dias após o transplante para o sistema hidropônico. As plantas foram coletadas e conduzidas ao laboratório, lavadas em água destilada e divididas em folhas, caules e raízes.

Em laboratório, a área foliar (AF) foi mensurada com um integrador de área foliar (Li-Cor, modelo Li-3100C). Em seguida, os materiais vegetais foram acondicionados em sacos de papel, separadamente, e transferidos para estufa com circulação forçada de ar, para determinação da massa seca de folha (MSF), massa seca do caule (MSC), massa seca do fruto (MSFR) e massa seca da raiz (MSR). Além disso, foram calculadas a massa seca da parte aérea (MSPA), massa seca total (MST) e a relação raiz parte aérea (RAIZ/PA).

Os dados obtidos foram submetidos à análise da variância, as médias comparadas pelo teste Tukey, a $1 \%$ de probabilidade através do programa estatístico SISVAR® 5.3 (FERREIRA, 2008).

\section{RESULTADOS E DISCUSSÃO}

Os dados de massa seca, para todas as partes da planta, apresentaram diferenças significativas a $1 \%$ entre os tratamentos aplicados (Tabela 1 ).

TABELA 1. Análise de variância para as variáveis de massa seca de plantas de $P$. angulata cultivadas em diferentes proporções de nitrato e amônio, Feira de Santana-Ba, 2018. 


\begin{tabular}{|c|c|c|c|c|c|c|c|c|c|}
\hline \multirow{2}{*}{ FV } & \multirow{2}{*}{ GL } & \multicolumn{8}{|c|}{ QUADRADO MÉDIO } \\
\hline & & MSR & MSC & MSF & MSFR & MST & MSPA & RAIZ/PA & $\mathbf{A F}$ \\
\hline TRAT & 3 & $4,55^{* *}$ & $74,35^{* *}$ & $38,92^{* *}$ & $38,89^{* *}$ & $530,16^{* *}$ & $443,02^{*^{*}}$ & $0,0035^{* *}$ & $24 \mathrm{e}^{-6^{* *}}$ \\
\hline ERRO & 32 & 0,63 & 6,32 & 3,28 & 4,86 & 44,59 & 35,60 & 0,0004 & $1,6 e^{-6}$ \\
\hline CV (\%) & & 35,49 & 28,53 & 27,44 & 46,02 & 29,73 & 29,52 & 18,09 & 30,57 \\
\hline
\end{tabular}

Massa seca da raiz (MSR); massa seca do caule (MSC); massa seca das folhas (MSF); massa seca dos frutos (MSFR); massa seca total (MST), massa seca da parte aérea (MSPA), razão raiz/parte aérea (RAIZ/PA) e área foliar $(\mathrm{AF}) .{ }^{* *}=$ significativo a $1 \%$ de probabilidade pelo teste $\mathrm{F}$.

As proporções de $\mathrm{NO}_{3}{ }^{-}: \mathrm{NH}_{4}{ }^{+}$de 100:0; 75:25 e 50:50 não diferiram estatisticamente (Tabela 2), demonstrando que as plantas obtiveram desempenho regular quando adicionados até $50 \%$ de amônio na solução nutritiva. Segundo Oliveira et al. (2007), os tratamentos com $\mathrm{NH}_{4}{ }^{+}$e $\mathrm{NO}_{3}{ }^{-}$balanceados disponibilizam mais $\mathrm{N}$, o que pode elevar a produtividade das culturas. Ohse et al. (2017) demonstraram que a alface apresentou incremento na massa seca com a utilização da proporção 90:10 de $\mathrm{NO}_{3}{ }^{-}: \mathrm{NH}_{4}{ }^{+}$, porém, sem grandes diferenças dos valores obtidos quando do uso das proporções 100:0 e 80:20. Barbosa et al. (2005), trabalhando com cinco variedades de crisântemo e cinco proporções de nitrato e amônio, observaram uma redução na massa seca da planta de $4,13 \%$ até $21,04 \%$ a depender da variedade.

O tratamento com maior porcentagem de amônio proporcionou redução em todos os caracteres avaliados com relação aos demais tratamentos, indicando uma preferência de absorção do nitrogênio na forma de nitrato pelas plantas de $P$. angulata. Foi verificada a redução de $58,76 \%$ no rendimento de massa seca total da planta quando comparada com o tratamento sem adição de amônio (Tabela 2), o que pode ser atribuído ao fato do íon $\mathrm{NH}_{4}{ }^{+}$causar toxidez em células vegetais, onde atua como desacoplador entre o fluxo de elétrons, fosforilação oxidativa e a fotofosforilação (TAIZ et al., 2017).

Por ser uma espécie com grande potencial para a fruticultura, a MSFR da $P$. angulata é uma variável de grande importância, e pôde ser observado que o cultivo com predomínio da forma nítrica proporcionou maiores valores (Tabela 2), indicando um maior número de frutos e/ou frutos de maior tamanho. Antúnez-Ocampo et al. (2014) observaram, em Physalis peruviana, que a aplicação conjunta de nitrato e amônio (50:50) aumentou o rendimento dos frutos, porém reduziu o teor de sólidos solúveis totais ( ${ }^{\circ}$ Brix).

Assim como as demais variáveis de massa seca, o valor de MSPA obtido foi bem inferior no tratamento com predomínio da fonte amoniacal. $\mathrm{O}$ amônio pode ter ação negativa sobre a condutância estomática da planta, interferindo diretamente na transpiração e fotossíntese e assim comprometendo o crescimento (SILVA et al., 2010), e esse menor incremento da MSPA refletiu na maior relação RAIZ/PA obtida no tratamento com $75 \%$ de amônio (Tabela 2).

TABELA 2. Médias das variáveis de massa seca de plantas de $P$. angulata cultivadas em diferentes proporções de nitrato e amônio, Feira de Santana-Ba, 2018.

\section{PROPORÇÕES $\mathrm{NO}_{3}{ }^{-} \mathrm{NH}_{4}^{+}$}




\begin{tabular}{ccccc} 
& $\mathbf{1 0 0 : 0}$ & $\mathbf{7 5 : 2 5}$ & $\mathbf{5 0 : 5 0}$ & $\mathbf{2 5 : 7 5}$ \\
\hline MSR (g) & $2,9666 \mathrm{a}$ & $2,6233 \mathrm{a}$ & $2,0411 \mathrm{ab}$ & $1,3433 \mathrm{~b}$ \\
MSC (g) & $10,5611 \mathrm{a}$ & $10,9400 \mathrm{a}$ & $9,0922 \mathrm{a}$ & $4,6744 \mathrm{~b}$ \\
MSF (g) & $7,8277 \mathrm{a}$ & $8,0022 \mathrm{a}$ & $7,0388 \mathrm{a}$ & $3,5488 \mathrm{~b}$ \\
MSFR (g) & $5,9444 \mathrm{a}$ & $6,0244 \mathrm{a}$ & $5,5144 \mathrm{a}$ & $1,6944 \mathrm{~b}$ \\
MST (g) & $27,3022 \mathrm{a}$ & $27,5888 \mathrm{a}$ & $23,6866 \mathrm{a}$ & $11,2588 \mathrm{~b}$ \\
MSPA (g) & $24,3355 \mathrm{a}$ & $24,9655 \mathrm{a}$ & $21,6466 \mathrm{a}$ & $9,9155 \mathrm{~b}$ \\
RAIZ/PA & $0,1222 \mathrm{ab}$ & $0,1033 \mathrm{bc}$ & $0,0922 \mathrm{c}$ & $0,1366 \mathrm{a}$ \\
AF (mm ${ }^{2}$ ) & $4617,45 \mathrm{a}$ & $5712,25 \mathrm{a}$ & $4769,45 \mathrm{a}$ & $1886,81 \mathrm{~b}$
\end{tabular}

Médias de tratamentos seguidas por mesma letra na linha não diferiram entre si pelo teste de Tukey a $5 \%$ de probabilidade. Massa seca da raiz (MSR); massa seca do caule (MSC); massa seca das folhas (MSF); massa seca dos frutos (MSFR); massa seca total (MST), massa seca da parte aérea (MSPA), razão raiz/parte aérea (RAIZ/PA) e área foliar (AF).

As maiores médias para AF foram obtidas quando utilizados 25 e $50 \%$ de amônio na solução nutritiva, apesar destes não diferirem do tratamento somente com a aplicação de nitrato, estando relacionados com a menor MSF obtidas para esses mesmos tratamentos. O nitrogênio é o principal nutriente que influência a área foliar e, consequentemente, a taxa fotossintética, promovendo maior acúmulo de massa seca nas plantas (MARSCHNER, 2012). Quando a fonte nitrogenada não é adequada, as plantas têm a absorção comprometida, apresentando desempenho semelhante ao déficit desse elemento.

Trabalhos com outra espécie do gênero, a $P$. peruviana, corroboram os resultados obtidos nesse trabalho. Antúnez-Ocampo et al. (2014) observaram que o cultivo com a combinação de nitrato e amônio proporcionaram maior altura e diâmetro do caule, sem prejuízos com a utilização do nitrato somente, e AntúnezOcampo et al. (2016) relataram, também, que a combinação das duas formas de nitrogênio favorecem a floração e a frutificação. Castañeda-Salinas et al. (2013) descrevem que a forma de nitrogênio preferencial para o cultivo da $P$. peruviana é a nítrica, porém a forma amoniacal pode ser utilizada sem prejuízo ao crescimento desde que inferior a $50 \%$, resultado semelhante ao obtido nesse experimento com a $P$. angulata, indicando uma similaridade entre as espécies do gênero quanto a absorção do nitrogênio.

\section{CONCLUSÃO}

As plantas de $P$. angulata utilizam preferencialmente o nitrogênio na forma nítrica, e o uso de solução nutritiva com maior quantidade de amônio reduz o acúmulo de massa seca nas plantas.

As proporções com $0 \%, 25 \%$ e $50 \%$ de amônio não interferiram na produção de massa seca de todas as partes das plantas de $P$. angulata $L$, podendo ser utilizada até $50 \%$ de amônio na solução nutritiva para o cultivo da espécie.

\section{REFERÊNCIAS}

ANTÚNEZ-OCAMPO, O. M.; SANDOVAL-VILLA, M.; ALCÁNTAR-GONZÁLEZ, G.; SOLÍS-MARTÍNEZ, M. Aplicación de amonio y nitrato en plantas de Physalis 
peruviana L., Agrociência, México, v.48, n.8, p.805-817, 2014. Disponível em: < http://www.scielo.org.mx/pdf/agro/v48n8/v48n8a4.pdf> Acesso em: 3 de abril de 2018.

ANTÚNEZ-OCAMPO, O.; SANDOVAL-VILLA, M.; ALCÁNTAR-GONZÁLEZ, G.; ALVARADO-LÓPEZ, J.; SABINO-LÓPEZ, E. Agrociência, México, v.50, n.5, p.603615, 2016. Disponível em: <http://www.scielo.org.mx/scielo.php? pid=S140531952016000500603\&script=sci_arttext> Acesso em: 03 de abril de 2018.

BARBOSA, J. G.; MUNIZ, M. A; MARTINEZ, H. E. P.; LEITE, R. A.; CARDOSO, A. A.; BARBOSA, M. S. Concentração de macronutrientes em crisântemo de vaso, cultivado sob diferentes relações $\mathrm{NO}_{3}{ }^{-} / \mathrm{NH}_{4}{ }^{+}$. Acta Scientia Agronomy, Maringá, v.27, n.3, p.387-394, $2005 . \quad$ Disponível em: <http://periodicos.uem.br/ojs/index.php/ActaSciAgron/article/view/1395/813>.

BITTSÁNSZKY, A.; PILINSZKY, K.; GYULAI, G.; KOMIVES, T. Overcoming ammonium toxicity. Plant Science, Shannon, v. 231, p. 184-190, 2015. Disponível em: <https://doi.org/10.1016/j.plantsci.2014.12.005> DOI: 10.1016/j.plantsci.2014.12.005.

CASTAÑEDA-SALINAS, C.; SANDOVAL-VILLA, M,; SÁNCHEZ-MONTEÓN, A. L.; ALEJO-SANTIAGO, G.; JIMÉNEZ-MEZA, V. M.; ABURTO-GONZÁLEZ, C. A.; GARCÍA-LÓPEZ, M. Respuesta de plántulas de uchuva (Physalis peruviana L.) a diferentes concentraciones de nitrato y amônio. Revista Bio Ciencias, Nayarit, v.2, n.3, p.148-153, 2013. Disponível em: <http://revistabiociencias.uan.mx/index.php/BIOCIENCIAS/article/view/45/43> Acesso em: 03 de abril de 2018.

CHANG, A.C.; YANG, T.Y.; RISKOWSKI, G.L. Changes in nitrate and nitrite concentrations over $24 \mathrm{~h}$ for sweet basil and scallions. Food Chemistry, v.136: p.955-960, 2013. Disponível em: <http://dx.doi.org/10.1016/j.foodchem.2012.08.084> DOI: $10.1016 /$ j.foodchem.2012.08.084.

CRUZ, J. L.; SOUZA FILHO, L.F.S.; PELACANI, C.R. Influência da adubação fosfatada sobre o crescimento do camapu (Physalis angulata L.). Revista Brasileira de Plantas Medicinais, Campinas, v.17, n.3, p.360-366, 2015. DOI: 10.1590/1983084X/13_060. Disponível em: <http://dx.doi.org/10.1590/1983-084X/13_060> DOI: 10.1590/1983-084X/13_060.

ESTEBAN, R.; ARZ, I.; CRUZ, C.; MORAN, J. F. Mechanisms of ammonium toxicity and the quest for tolerance. Plant Science, Shannon, v. 248, p. 92-101, 2016. Disponível em: <https://doi.org/10.1016/j.plantsci.2016.04.008> DOI: 10.1016/j.plantsci.2016.04.008

FERREIRA, D. F. SISVAR: Um programa para analises e ensino de estatística. Revista Científica Symposium,v. 6, p. 36-41, 2008.

HUANG, L.; LU, Y.; GAO, X.; DU, G.; MA, X.; LIU, M.; GUO, J.; CHEN, Y. Ammonium induced oxidative stress on plant growth and antioxidative response of duckweed (Lemna minor L.) Ecological Engineering, Amsterdam, v. 58, p. 355-362, 
2013. Disponível em: <https://doi.org/10.1016/j.ecoleng.2013.06.031> DOI: 10.1016/j.ecoleng.2013.06.031.

LEITE, R. S.; TANAN, T. T.; NASCIMENTO, M. N.; OLIVEIRA, L. M.; ABREU, P. A. S. Hydroponic cultivation of Physalis angulata L.: growth and production under nitrogen doses. Pesquisa Agropecuária Tropical, Goiânia, v. 47, n. 2, p. 145-151, Apr./Jun. 2017. Disponível em: <http://dx.doi.org/10.1590/1983-40632016v4744652> DOI: 10.1590/1983$40632016 \mathrm{v} 4744652$.

LIMA, C. S. M., SEVERO, J., ANDRADE, S. B., AFFONSO, L. B., ROMBALDI, C., \& RUFATO, A. R. (2013). Qualidade pós-colheita de physalis sob temperatura ambiente e refrigeração. Revista Ceres, 60(3), 311-317. Disponível em: <http://dx.doi.org/10.1590/S0034-737X2013000300002>ＤOI: 10.1590/S0034$737 X 2013000300002$.

MARSCHNER, P. Marschner's mineral nutrition of higher plants. 3. ed. San Diego: Academic Press, 2012. 672 p.

MARTÍNEZ-ANDÚJAR, C.; GHANEM, M. E.; ALBACETE, A.; PÉREZ-ALFOCEA, F. Response to nitrate/ammonium nutrition of tomato (Solanum lycopersicum L.) plants overexpressing a prokaryotic $\mathrm{NH}_{4}{ }^{+}$- dependente asparagine synthetase. Journal of Plant Physiology, v. 170, p. 676-678, 2013. Disponível em: <https://doi.org/10.1016/j.jplph.2012.12.011> DOI: 10.1016/j.jplph.2012.12.011

MAROUELLI, W. A.; SOUZA, R. B.; BRAGA, M. B.; SILVA, W. L. C. Evaluation of sources, doses and application schedules of nitrogen on drip-irrigated tomato. Horticultura Brasileira, v. 32, p. 327-335, 2014. Disponível em: < http://dx.doi.org/10.1590/S0102-05362014000300015>DOI: 10.1590/S010205362014000300015.

OHSE, S.; DOURADO-NETO, D.; MANFRON, P. A.; OTTO, R. F.; GODOY, A. R. Rendimento e acúmulo de nitrato em alface hidropônica sob proporções de nitrato e amônio . Revista Campo Digit@I, v. 12, n. 1, p.52-64, jan./jul., 2017. Disponível em: <http://revista.grupointegrado.br/revista/index.php/campodigital/article/view/2321/93>

OLIVEIRA, L. A.; KORNDÖRFER, G. H.; PEREIRA, A. C. Acumulação de silício em arroz em diferentes condições de $\mathrm{pH}$ da rizosfera. Revista Brasileira de Ciência do Solo, Viçosa, v.31, n.4, p.685-690, 2007. Disponível em: <http://dx.doi.org/10.1590/S0100-06832007000400009> DOI: 10.1590/S010006832007000400009.

SARASKETA, A.; GONZÁLEZ-MORO, M. B.; GONZÁLEZ-MURUA, C.; MARINO, D. Exploring ammonium tolerance in a large panel of Arabidopsis thaliana natural accessions. Journal of Experimental Botany, Lancaster, v. 65, n. 20, p. 6023-6033, 2014. Disponível em: <https://doi.org/10.1093/jxb/eru342> DOI: 10.1093/jxb/eru342

SARRUGE, J.R. Soluções nutritivas. Summa Phytopathologica, Botucatu, v. 1, n. 3, p. 231-233, 1975. 
SILVA, P. C. C.; COUTO, J. L.; SANTOS, SANOS, A. R. Efeito dos íons amônio e nitrato no desenvolvimento do girassol em solução nutritiva. Revista da Faculdade de Zootecnia, Veterinária e Agronomia, Uruguaiana, v.17, n.1, p. 104-114. 2010. Disponível

em

<http://revistaseletronicas.pucrs.br/ojs/index.php/fzva/article/view/7140/5456>

Acesso em: 03 de abril de 2018.

STEHMANN, J. R.; MENTZ, L. A.; AGRA, M. F.; VIGNOLISILVA, M.; GIACOMIN, L.; RODRIGUES, I. M. C. 2015. Solanaceae. In: Lista de Espécies da Flora do Brasil. Jardim Botânico do Rio de Janeiro. Disponível em: <http://floradobrasil.jbrj.gov.br/jabot/floradobrasil/FB225> Acesso em: 02 de março 2018.

STEFEN, D. L. V.; SOUZA, C. V. COELHO, C. M. M.; GUTKOSKI, L. C.; SANGOI, L. $A$ adubação nitrogenada durante o espigamento melhora a qualidade industrial do trigo (Triticum aestivum cv. Mirante) cultivado com regulador de crescimento etiltrinexapac. Revista de la Facultad de Agronomía, La Plata (2015) Vol 114 (2): 161-169.

Disponível

em:

<http://revista.agro.unlp.edu.ar/index.php/revagro/article/view/28/273>

TAIZ, L.; ZEIGER, E.; MOLLER, I.M. et al. Fisiologia e desenvolvimento vegetal. 6. ed. Porto Alegre: Artmed, 2017. 